\title{
From telework project planning to project co-ordination, an integrated approach
}

\author{
José Augusto Pinho Silva ${ }^{1}$; João José Pinto Ferreira ${ }^{2}$ \\ ${ }^{1} M S C$ Student at FEUP-DEEC \\ ${ }^{2}$ FEUP-DEEC / INESC-UESP \\ $R$. José Falcão 110, 4000 Porto, Portugal \\ Phone: +351 220943 00; Fax.: + 35122008487 \\ E-mails: jasilva@usa.net; jipf@fe.up.pt; \\ URL: http://www.fe.up.pt/ jjpf/
}

\begin{abstract}
This paper focuses on the telework support to the so-called Small Informationbased Organisations, where all activities involve information processing and transfer, usually among sub-contracted teleworkers. Most of these companies run by managing multiple parallel projects, i.e. one-of-a-kind business processes, each project usually involving remote activity execution by teleworkers. There is therefore a clear demand for workflow management systems supporting telework, featuring facilities that further enhance traditional office automation systems by filling the required support to geographically distributed co-operative work. The work presented was undertaken during a telework workflow management system requirements' specification, to support the so-called Small Information-based Organisations. To this end, we analysed the virtual enterprise life cycle, identifying the required modelling tool types for each of the required steps. The consecutive project steps from project planning to co-ordination were then identified, with special relevance to the definition of the so-called execution primitives and to the support of human interaction through the speech-act theory. This approach allowed the description of a consistent life-cycle approach from project planning to coordination.
\end{abstract}




\section{INTRODUCTION}

Telework is currently considered as an innovative work organisation-form for new decentralised organisational structures, demanding the use of workflow management technology. The flexibility of telework in the dimensions of time, location of task execution and the legal status of employment contracts will pave the way to the companies' competitive advantage.

This paper focuses on the telework support to the so-called Small Informationbased Organisations, where all activities involve information processing and transfer, usually among sub-contracted teleworkers. Previous attempts made by these companies to automate office work and increase productivity failed, because individual activities were automated without an understanding of how those activities fitted into the entire business processes. The fact is that most of these companies run by managing multiple parallel projects, i.e. one-of-a-kind business processes, each project usually involving remote activity execution by teleworkers. There was therefore a clear demand for the use of workflow management technology, which takes the formal part of procedure processing applications and combines it with general communication and information sharing facilities. As a result, workflow management systems for telework should further enhance traditional office automation systems by filling the required support to geographically distributed co-operative work.

This paper presents the work undertaken during a telework workflow management system requirements' specification, to support the so-called Small Information-based Organisations. In this context, we had in mind the three typical entities involved in such an endeavour: the project planner, the project manager and co-ordinator and the teleworker. We start by analysing the actual virtual enterprise life cycle, identifying each of the involved steps, and the required modelling tool types for each one of those steps. We finally describe the approach taken in the integration of the identified consecutive project steps from project planning to co-ordination, with special relevance to the execution primitives definition and support to human interaction through the speech-act theory.

\section{LIFE-CYCLE ASPECTS}

Telework may be included within the scope of the distributed / virtual enterprise. As a result, it could be described by buzzwords such as co-ordination, distribution and integration. We aim indeed at solving the problem of information and processing distribution across geographically far apart enterprise working places, allowing for the co-ordination of distributed information processing activities.

The engineering of the virtual enterprise may follow the Purdue Enterprise Reference Architecture (PERA) [Williams, Hong] life cycle, as illustrated in Figure 1. The life-cycle starts with Identification phase of the Enterprise Business Entity, leading to the first description of the management's mission, vision and values for that entity plus any further philosophies of operation or mandated actions concerning it, such as choice of processes, vendor selection, etc. By the end 
of this phase, we should have a clear view of what we want to do, e.g. "We want to build an interactive presentation of the Portuguese History through the last century, by having the co-operation of the best specialists around the world. This job will be accomplished by using the latest communication and project management technologies to co-ordinate the tasks in the different activities and to save on travelling expenses".

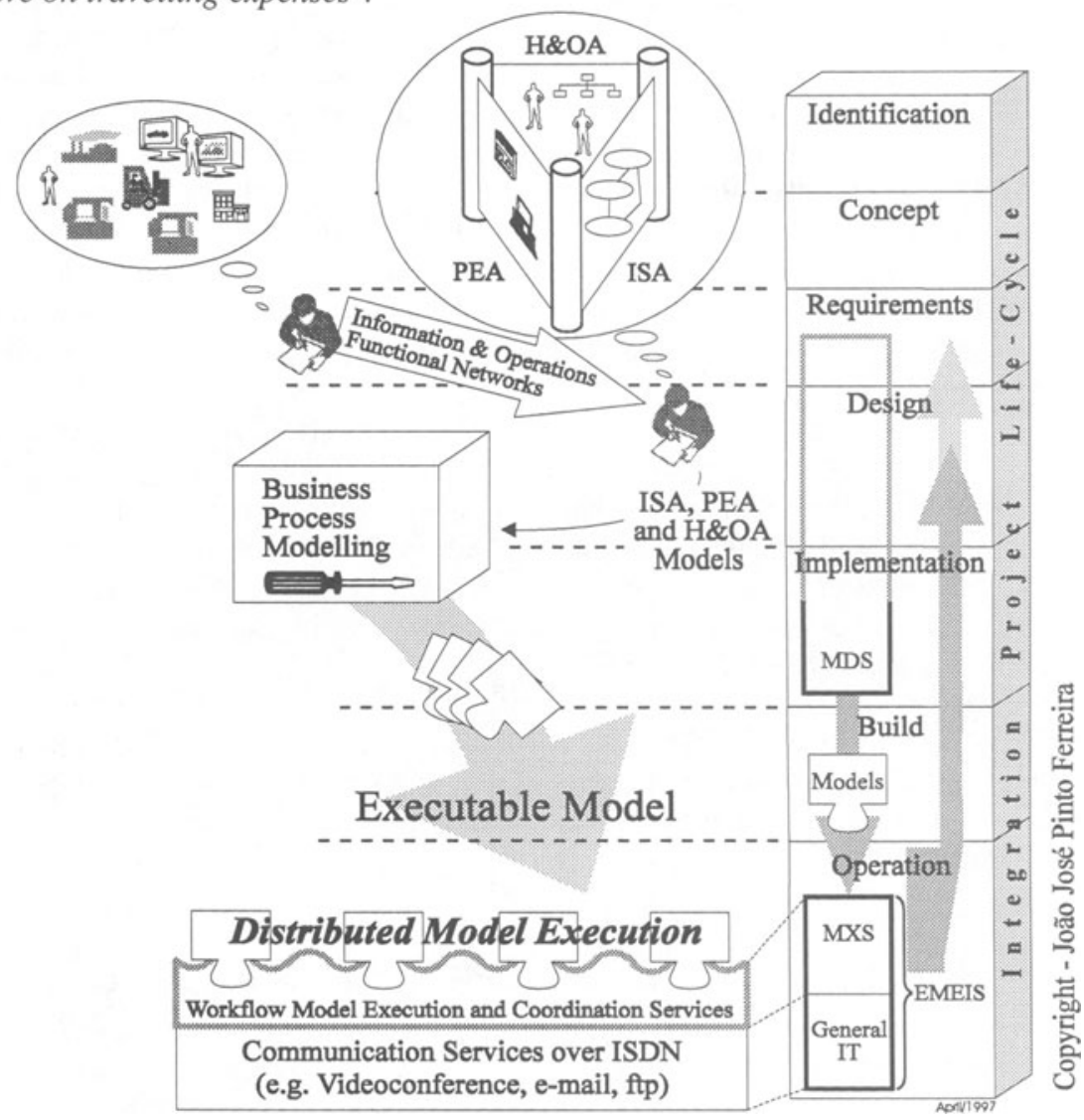

\section{Figure 1 - Telework Engineering Life-cycle.}

From the management mission, vision, etc., the operational policies for the units for all areas of potential concern are derived in the Concept Phase. The earlier prescription and selection by the management of possible options leads to the establishment of operational requirements for the project, which in turn lead to a statement of the requirements for all equipment and for all methods of operation which are developed along the Definition Phase. It should finally be noted that these are only two types of requirements, those defining information processing tasks and those defining physical operation tasks. These tasks become collected into modules or functions, and these can in turn be connected into networks of information or of material, resulting in the Information Functional Network or in 
the Operations Functional Network (Figure 1). These networks are in fact describing the information flow and the actual task network, which could be represented as an activity network (e.g. PERT network - Program Evaluation and Review Technique), having in mind the required resources and their geographical position. This completes the so-called design phase.

No consideration of the place of the humans in the system has been made yet. PERA nevertheless recognises that in any enterprise development program, the human and organisational system is as important as the information system and physical components. This means that, once the implementation is considered, the first need is to define which tasks on either side of the overall architecture people will fulfil. Accordingly, three Implementation or Physical Architectures are introduced into the telework-engineering project. These are:

- the Information System Architecture (ISA in Figure 1), (comprising the description of all infrastructure and application software, e.g. workflow management system, videoconference software, etc., as well as their integration);

- the Human and Organisational Architecture (H\&OA in Figure 1), (encompassing the role played by teleworkers in remote working places, e.g. teleworker and/or co-ordinator, as well as their integration into the overall organisation so that enterprise objectives can be achieved);

- And the Physical Equipment Architecture (PEA in Figure 1), (referring to the actual physical system architecture, such as computing, networking and live communication equipment).

In the design phase all these aspects should be considered, and this means that our modelling language should be able to take the detailed PERT project network, including detailed information regarding the required information flow. This requires a further model refinement which will lead to the construction of the socalled executable model.

\section{FROM PROJECT PLANNING TO PROJECT COORDINATION}

\subsection{Project Management}

In this paper, we are concerned with those companies running their businesses by managing multiple parallel projects, e.g. one-of-a-kind business processes. Most of these companies use project management tools supporting techniques such as PERT (Program Evaluation and Review Technique) and/or CPM (Critical Path Method) [Taha]. These techniques require the definition of the project activities and their attributes (time, cost and resources); the activity execution sequence; and how can they be executed in a optimised and competitive way. Project Planning deals fundamentally with problems involving minimisation of time and cost, while dealing with resource constraints. These methods usually comprise the following 3 phases: Planning, Schedule and Control. The Planning phase begins with the subdivision of the project into different activities, each one with an estimated execution time, allocated resource and expected cost. An arrow (network) diagram 
is then built, with each arrow representing an activity. The whole diagram is therefore the graphic representation of the project activities' interdependencies. Follows the scheduling phase whose main objective is the construction of a time chart showing the start and conclusion of each activity. In this phase, critical activities that require special attention to complete the project as planned are identified. On the other hand, for non-critical activities, the scheduler will further define items such as time tolerances and acceptable delays. Conventional project control follows the so-called scheduling/control phase. Based in the previously presented time charts and in the arrow diagrams, progress reports are produced during the Control phase. So that, fast reaction to unforeseen events can be achieved by activity re-scheduling, the overall project information must updated and analysed as frequently as required. Project Control is therefore performed by the project manager based on the analysis of consistent updates made to the project activity chart. The effectiveness of this approach replies mainly on the project manager ability to communicate with the different human resources involved in the project, as well as its ability to adequately use available communication channels such as Fax, Phone and E-mail.

The ever-growing telework popularity, along with the working place geographic distribution, and teleworker time flexibility, clearly resulted in the increased demand for enhanced communication and co-ordination facilities for telework, usually not provided by conventional project management tools. Improved project management may be achieved by using workflow management tools, providing the adequate means for the distributed model-based co-ordination, achieved through the actual activity model enactment. To this end, the next paragraphs briefly present the model execution technique used within the project.

\subsection{Model enactment}

Figure 1 illustrates the so-called Enterprise Model Execution and Integration Services (EMEIS). The EMEIS upper layer defines a set of services allowing the interpretation of an activity model (MXS - Model execution services). This model having been previously developed in a Model Development Environment, which makes use of the Model Development Services, (MDS). The EMEIS further includes the general IT services, allowing for the required distribution and multimedia communication among geographically distributed working places.

Our approach provides the user with the very easy and user-friendly project management user interface of Pert-Networks or Gantt charts. However, these lack the needed executable primitives, capable of supporting automatic model execution. Model execution automation for execution (performed by the EMEIS Model execution services) demands in fact for the actual model formal description. To this end, we used the so-called "move" and "produce" primitives based on the [Bauer] production activity control (PAC) model.

Figure 2 illustrates the PAC model, which is being applied widely in the discrete manufacturing area. This model foresees that any plan operation can be decomposed into a "Move" or "Produce" primitive. In this paper, we apply this principle arguing that Telework is mainly a distributed information processing 
activity, and therefore, a Telework activity plan can be decomposed as well into the execution of "Move" (information transfer) or "Produce" (information processing according to a defined specification) primitives. In fact, Teleworkers will mainly produce documents in the electronic format (according to a particular specification), which are successively moved along the project activity network resources (teleworkers). This picture further illustrates the monitoring facility, providing the dispatcher module with the required updated information to drive the actual model execution. On the other hand, compared to conventional human-based activity control, monitoring information may be used to react faster to unforeseen events allowing early warnings and activity re-scheduling.

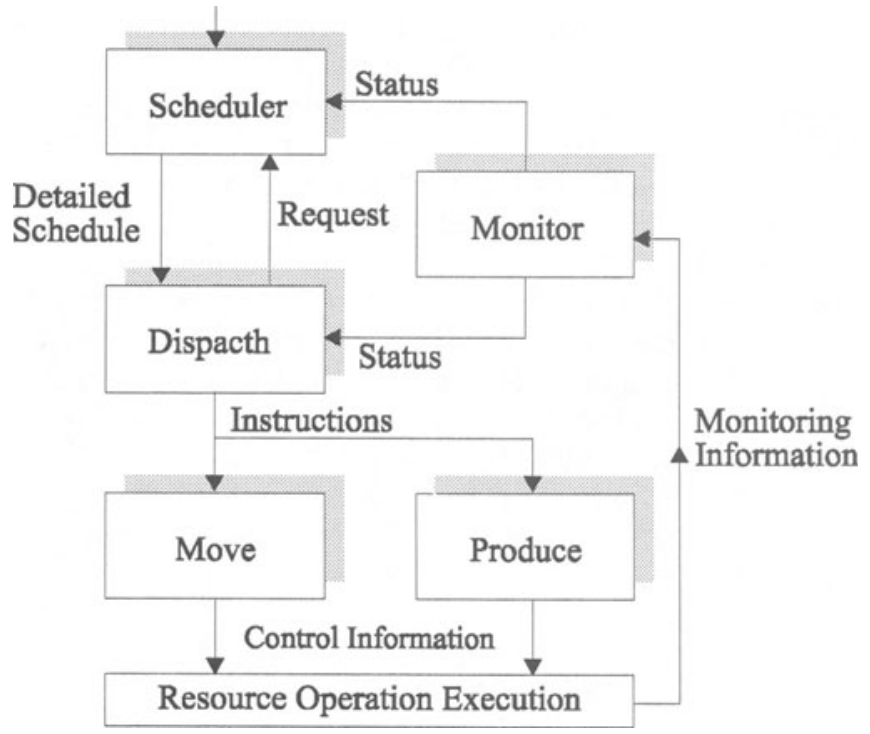

Figure 2 - Shop Floor Control Model.

\subsection{Incorporating human resource interaction into the model execution}

The main issue now is the adequate support of Teleworkers interaction. Teleworkers are human operators, and the success of telework co-ordination tools is strictly bound to the satisfactory support of collaboration among the project telework elements.

[Schäl] defines the existence of a customer/supplier relationship as the main aspect of business processes, further arguing that customer satisfaction in such customer/supplier relations should be seen as a linguistic expression. This communicative dimension should therefore be incorporated in the workflow management systems by supporting communication and co-operation among teleworkers in the "language/action perspective". The "language/action perspective" is described in the traditional framework of linguistic theory (syntax, semantics and pragmatics), and is based on "speech-act theory". The most 
important aspect of "speech-acts" is the conveyed speakers' intention, rather than its form or textual meaning. Speech acts exchanged between two partners constitute conversations, which can be distinguished by four types: conversation for $\sim$ action, $\sim$ possibility, $\sim$ clarification, and $\sim$ orientation.

Our approach uses the so called "Four-step Action Workflow model" [Schäl], defined as a simplified version of the conversation for action. As illustrated in the Figure 3, in the first phase (request), the customer asks for a service or product. In the second (commitment), the supplier promises to fulfil a specific condition. In the third (performance), the supplier fulfils his work which leads to the delivery of the requested service or product. The final phase (evaluation), closes the loop and involves the customers acknowledgement or formal declaration of satisfaction.

As illustrated, the customer / supplier iteration loop for a specific service is only finished whenever customer satisfaction is reached. The picture represents a simple four-transition loop, request, promise, report and declare. However, new loops can emerge whenever needed, such as when a clarification (phase 1), negotiation (phase 2), delegation (phase 3) or confirmation (phase 4) is required. As an example, we could draw a scenario where the request move triggers a specific clarification action workflow.

1

Customer

asks for action

(request phase)

4

Customer

accepts report and

declares satisfaction

(evaluation phase)

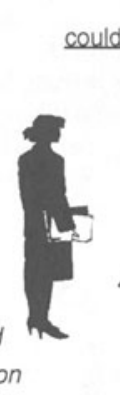

O.K. thank you

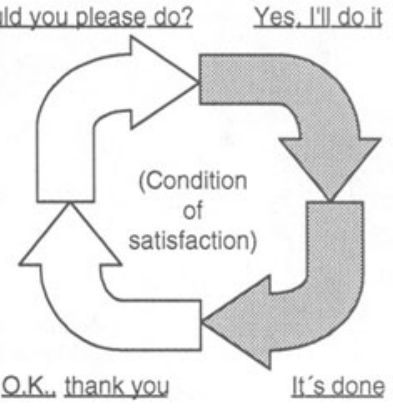

2

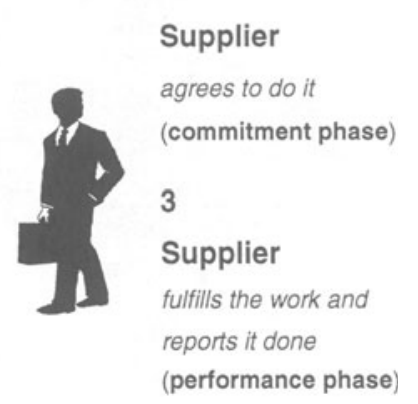

Figure 3 - Basic workflow model describing customer/supplier relations in the language/action perspective.

\subsection{The integrated solution}

Previous paragraphs guided us in the search of an integrated solution to supporting the project planning, scheduling/management and control through its complete life cycle. The path to the integrated solution is illustrated in Figure 4. The first step is clearly the use of conventional methods such as CPM/PERT, to model the project activity net. It is also clear that these tools provide the useful concepts for project planning, and this means that they should be used. As the entire project planning evolves, the initial PERT/CPM model is enriched with the so-called execution primitives "Move" and "Produce". These primitives will allow the construction of the executable model, to be used on the co-ordination of Telework activities. The "Move" primitive will allow the modelling of electronic document transfer 
between Teleworkers, whereas the "Produce" primitive will allow the detailed description of the task to be executed remotely by a particular Teleworker.

The final step needs no modelling effort, as it is embedded in the actual model execution platform at the planning, scheduling and execution phase. In the planning/scheduling phases, there are predefined dialogues supporting negotiation of task assignments of Teleworkers, therefore facilitating the communication during the initial project planning/scheduling tasks. During the execution phase there is an embedded support to communication between Teleworkers and the coordinator. Whenever required, the teleworker dialog support is automatically triggered by the execution of "Move" primitives, by embedded specific speech-acts from the "conversation for action" or other types of conversations, defined in the "speech act theory". These dialogs can also be triggered by any teleworker at any time for clarification, further negotiation or any other required action.

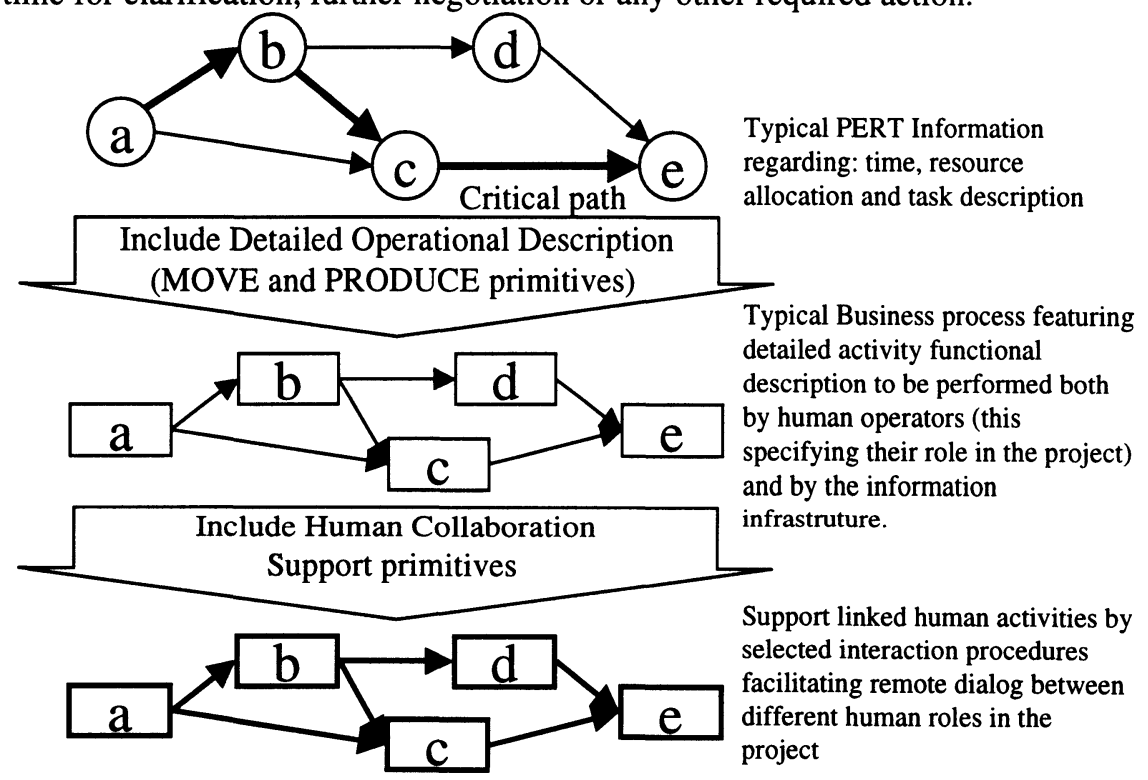

Figure 4 - Different steps towards Workflow the model construction.

\section{MODEL-DRIVEN ACTIVITY EXECUTION, EXAMPLE}

Figure 5 illustrates a network of six activities $(\mathrm{U}, \mathrm{V}, \mathrm{W}, \mathrm{X}, \mathrm{Y}, \mathrm{Z})$ under execution, driven by the workflow management system co-ordination engine. The inherent communication among teleworkers, and between them and the co-ordination engine is further illustrated by the action workflow loops pictured between the ongoing activities and the co-ordination engine. The centralised approach for coordination is also pictured, this meaning that all workflow interactions are performed through the co-ordination engine whose main features are: management and co-ordination of communications between activities/teleworkers; information 
flow management (filtering; warnings; routing; reporting; transferring; verifying; etc.).

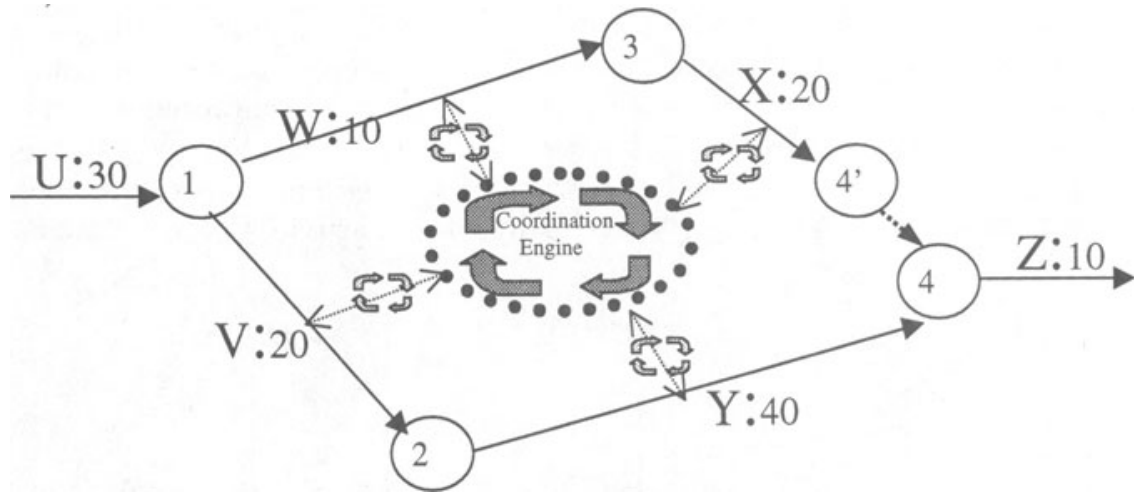

Figure 5 - Partial aspect of Pert net enriched with communication dimension.

The scenario described in this paragraph describes on-going transactions for a particular a window in time, triggered by the conclusion of activity $U$ which in turn triggers activities V and W that should then start and use U's outputs. We therefore focus our description in the last two phases of Activity U Action Workflow cycle (report and evaluate), and the first phases of activities $\mathrm{V}$ and $\mathrm{W}$ action-workflow cycle (request and promise).

In our approach, we aimed at achieving an environment fostering an highly controlled communication between activity performers, i.e. teleworkers. To this end, we argue that minimum erroneous communication interpretations can be achieved by defining patterns of communications, available through the use of templates. This strategy is further allowing the automatic supervision and monitoring without the project managers' intervention. In the course of a particular dialog context, these communication templates are suggested to the teleworker as possible expected replies (action workflow move) in response to incoming messages. System imbedded speech-acts templates selected from the "conversation for action" are in fact automatically triggered by the execution of the activity model "Move" primitives.

Our scenario (Figure 6) starts with transfer of the final report carrying an attached file containing the results of the work (Performance Phase) executed during activity $\mathbf{U}$, using the template Report Communication with code (RP-1). As the Co-ordination Engine (CE) receives that information, it checks the schedule and identifies that the following activities are $\mathbf{V}$ and $\mathbf{W}$, and that they have the results of $\mathrm{U}$ as inputs. The $\mathrm{CE}$ immediately sends a Declarative Request Communication (D-RQ-1) to $\mathbf{V}$ and $\mathbf{W}$, with the attached file received from $\mathbf{U}$ asking for acceptance. In our example we illustrate two different replies: a) $\mathbf{V}$ accepts (D-PR-2), and b) W refuses and counter-offers by stating its own reasons for that reply (D-CT-1,2). As the CE receives the counter-offer, it immediately sends an information to activity $\mathbf{V}$ and $\mathbf{W}$ stating that the incoming data was refused (D-WD-1) and therefore should be disregarded. At the same time the CE sends activity $\mathbf{U}$ the refuse information and the reasons, asking for possible 
modification (DL-1,2,3). After reviewing and correcting the work, activity $\mathbf{U}$ sends once more its results and asks for acceptance (RP-2,1), which is again forwarded for activities $\mathbf{V}$ and $\mathbf{W}$ (D-RQ-1). Finally, the acceptance confirmation from both activities $\mathbf{V}$ and $\mathbf{W}$ (D-PR-2) is received. As the CE receives the confirmations, it is clear that activity $\mathbf{U}$ has completed its job successfully. As a result a positive evaluation (EV-1) accepting the work is sent to activity $\mathbf{U}$, and requests (RQ-1 and RQ-2) are sent to $\mathbf{V}$ and $\mathbf{W}$, which in turn reply by promising to start and do their jobs (PR-1 and PR-2). The code (EV-2) finally informs activity $\mathbf{U}$ of conclusion of its work.

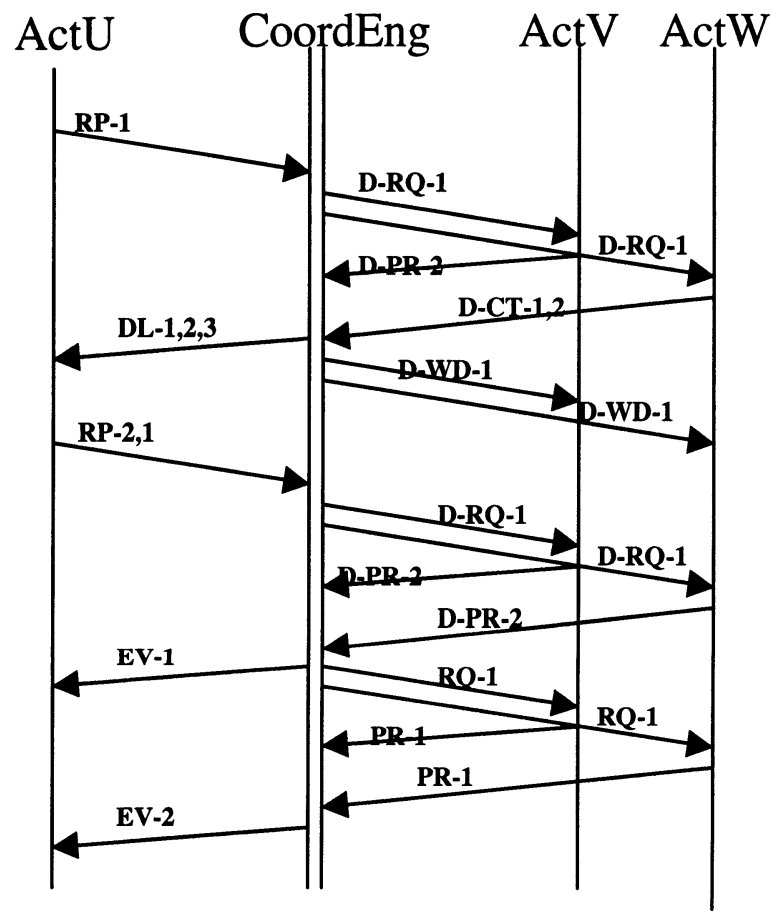

Figure 6 - Communication scenario message sequence chart.

Other scenarios could be defined and described, illustrating the use of other speech-acts templates triggered either by the "move" primitives, or by the teleworker in the course of its task execution, such as speech-acts for further action, clarification, orientation or possibility.

\section{CONCLUSIONS}

This paper focused on the telework support to the so-called Small Informationbased Organisations, where all activities involve information processing and transfer, usually among sub-contracted teleworkers. We started by identifying lifecycle requirements as well as the use of the activity model enactment concept 
applied to project management. This first approach was then followed by the presentation of the rationale underlying the selection of the "move" and "produce" primitives for model enactment. As an innovative work organisation-form for new decentralised organisational structures, telework is particularly demanding in the support to structured collaboration among teleworkers. This lead us to the use of workflow management technology embedding the so-called communicative business process dimension, by incorporating in the workflow management systems co-operation among teleworkers in the "language/action perspective". The "Four-step Action Workflow model" defined as a simplified version of the conversation for action, was then suggested and a strategy for its implementation briefly presented. As future work, we foresee further elaboration on the conversation templates for typical telework activities, as well as its test in laboratory for worst case scenarios.

\section{REFERENCES}

[Williams] T.J.Williams (1993): The Purdue Enterprise Reference Architecture (B14), H. Yoshikawa and J. Goossenaerts (Editors), Elsevier Science B.V. (North-Holland) 1993 IFIP

[Hong] Hong Li (1994): A formalization and extension of the Purdue Enterprise Reference Archhitecture and the Purdue Methodology, A Thesis Submitted to the Faculty of Purdue University by Honh Li, In partial fulfillment of the requirements for the Degree of Doctor in Philosophy, December, 1994

[Ferreira] J. J. Pinto Ferreira, Ângelo Martins, Wilhelm Dangelmaier, Stephan Kress, Thomas Goletz (1998): "Telework Business Process Co-ordination The supporting tool engineering life cycle" BASYS'98 is 3rd IEEE/IFIP International Conference on Information Technology for BALANCED AUTOMATION SYSTEMS in Manufacturing. BASYS'98 held in Prague, Czech Republic, from 26 to 28 Aug 1998

[Bauer] Bauer et al (1994) Shop Control Systems: From Design to Implementation. Chapman \& Hall, ISBN 0-412-36040-3

[Schäl] Schäl, Thomas (1996) Workflow Management Systems for Process Organisations. Springer .

[Taha] Taha, Hamdy, A.(1984) Operational Research: An introduction. Prentice Hall International Ed.

\section{BIOGRAPHY}

José Augusto Pinho Silva, received his Engineering Degree in Electronics and Telecommunications in 1990 and a Post-Graduate Degree in Industrial Management in 1993 both by the Aveiro University in Portugal. In 1991 he joined for two years the Control and Programming Division of "TLP,SA" (Portugal Telecom). In 1994 joined the distribution enterprise "f.fonseca,Lda", (Industrial Automation Systems), working for one year with Mitsubishi Automation Products. 
Since 1995 he is working in the "IEFP/DN-FRM" managing a Professional Education pool. Since 1995 coursing the MSc on Electrical and Computers Engineering at the Engineering Faculty in Porto, Portugal. Since 1996 he is lecturing at "ISVOUGA" domains such as Electricity, Automation and Control. His research interests include Telework, Industrial Automation and CIM.

João José Pinto Ferreira is currently Assistant Professor at the Engineering Faculty of Porto Electrical and Computers Engineering Department where he has been lecturing since 1987. He is also researcher and project leader at INESC Production Systems Engineering Group in Porto. Born in August 1964, he got his Licenciatura degree by University of Porto in 1987, his MSc by the University of Porto in 1991 and his PhD also by University of Porto in 1995 all in Electrical and Computers Engineering. Since 1987 participated in several R\&D projects, and is currently the Project and Technical Co-ordinator of the COBIP project ("Telework co-ordination services for co-operative business processes" UR 4002 - SECTOR 6 of the TELEMATICS APPLICATIONS Programme). 